\title{
Nonprogramming laboratory assignments for the introductory AI course using the FLAIR system
}

\author{
J.D. Wilson \\ G. Ingargiola \\ R.M. Aiken \\ N. Hoskin \\ Temple University \\ Philadelphia \\ USA
}

\begin{abstract}
Undergraduate computer science students benefit from nonprogramming laboratory assignments which have them explore and experiment with algorithms and abstractions in a concrete learning environment. FLAIR, a repository of learning materials which supports the laboratory component of the introductory undergraduate AI course affords interesting nonprogramming assignments of this kind. In the following discussion the FLAIR system is described and three laboratory assignments which have been used with FLAIR, are presented.
\end{abstract}

Main conference themes: flexible learning

Educational areas: higher education

Study topics: computer science/informatics

Secondary keywords: courseware, learning systems, pedagogy, teaching materials 


\section{INTRODUCTION}

Research in learning supports the commonly held view that students learn abstractions more adequately if these abstractions are reinforced with concrete problem solving experiences (See, for example, [1] and [2]). Problem solving exercises in university undergraduate Computer Science (CS) courses have typically had students use a programming language to solve paradigmatic problems in the subject area. Although it is important to include programming problems in CS courses, the need to master the details of a programming language and frustration with the programming environment often undermine student interest in learning about underlying conceptual issues. On the other hand it is likely that students would benefit from a laboratory based learning environment in which they could explore aspects of a knowledge

FLAIR (Flexible Learning with an Artificial Intelligence Repository) was developed, in part, to provide such an environment for the undergraduate course in Artificial Intelligence (AI). In the following discussion we give an overview of the FLAIR system and describe three nonprogramming laboratory assignments designed to be used with FLAIR. All of these assignments are intended to help develop a better understanding of theoretical issues in AI by providing students with concrete problem solving experiences. The overview of FLAIR concentrates on those aspects of the system which are relevant to the carrying out of the assignments.

\section{THE FLAIR SYSTEM}

FLAIR is a repository of pedagogical materials designed to supplement lectures, programming and textbook exercises in an undergraduate AI course. Although FLAIR is intended for the AI course, its design principles can be generalized easily to other undergraduate CS courses. Additional details regarding the structure of the FLAIR Repository Framework can be found in [3].

We will confine our attention here to those features of FLAIR for which nonprogramming laboratory assignments have been designed and used; these include graphical navigators for exploring FLAIR's object database and animated visual environments (called 'modules') which allow the user to experiment with standard algorithms in various AI subdomains.

\section{Navigators}

The FLAIR repository is a collection of persistent, typed objects with attributes and relations. Objects may be shared (stored in a common area) or private. 
Examples of FLAIR objects are animated modules, knowledge domain concepts and algorithms in the form of pseudocode and Lisp programs.

Navigators display FLAIR objects as nodes in a two-dimensional graph or 'conceptual map' (see Fig. 1). Each node in the graph represents a FLAIR object and each link between two nodes represents a relation which exists between those two objects. The user may inspect any object represented in the graph. When inspecting an object the user may examine its properties, browse its text or hyperlink to related objects.

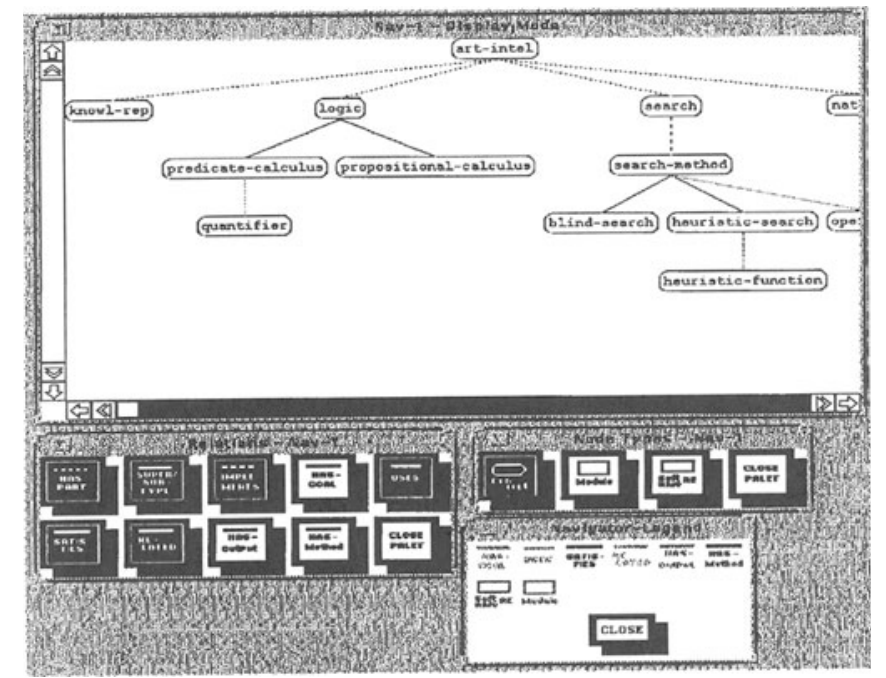

Fig. 1 The FLAIR Navigator

Hypertext browsers are common features in many systems whereas graphical navigators are relatively uncommon. The advantages of a graphical navigator are several: it provides a visual overview of the browsable domain and (potentially) of the paths taken through the domain; it allows the user to modify objects without losing sight of their context in the object network; and it permits the user easily to transition between distant parts of the conceptual map.

\section{Modules}

FLAIR Modules are highly visual and animated interactive environments which allow the student to experiment with various $\mathrm{AI}$ algorithms and techniques. 
Module environments consist of a number of concurrent displays which provide the user with a problem solving environment and informative messages.

Modules include features designed for specific algorithms and problem solving environments, but all share a set of kernel features. Standard display controls allow the user to adjust the execution speed, pause and single-step, or proceed through the algorithm. As the algorithm executes the intermediate results are displayed. In addition the user can activate several ancillary environments and displays including displays of the program code or the pseudocode for the (current) algorithm.

Modules can call each other. If during the operation of one module data must be processed by an algorithm which is present in another module, the user is given the option of exploring the operation of the called module concurrently with that of the calling module. In this manner the user can observe in detail how the called algorithm operates on the data. The theorem proving modules are interrelated in this way.

\section{The Map-Search module}

The Map-Search module in FLAIR allows a student to explore graphically the behaviour of several standard search algorithms on a geographical map (Fig. 2). The main map window provides a graphical overview of the search space and of the algorithm's progress.

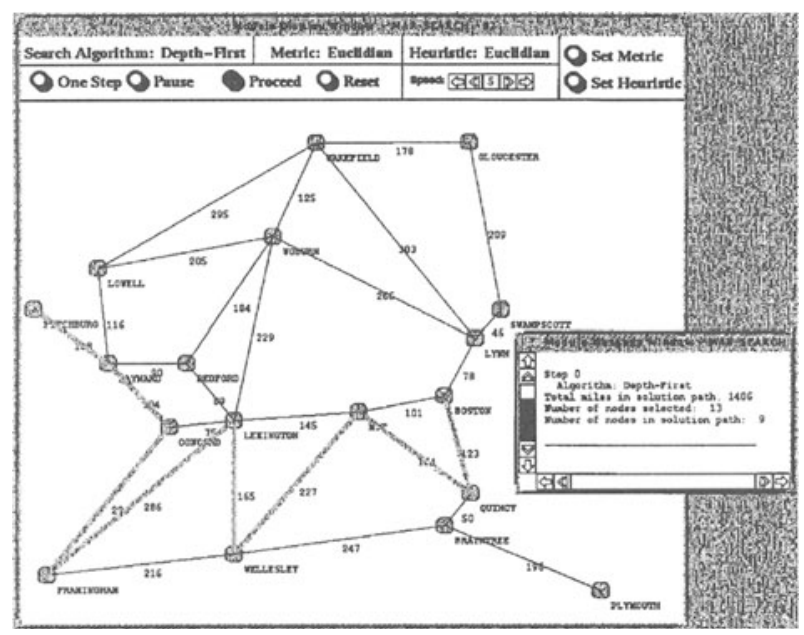

Fig. 2 The Map-Search module showing the main display window and message window 
The user specifies a start city and a goal city and selects a search algorithm (for example Hill Climbing or Best First) from a list of available algorithms. The current partial path is displayed on the map and the cities which have been examined are marked. A separate message window chronicles significant events which occur during each search, providing summary results such as the number of cities explored.

The user also can activate lower level displays which show the changing state of the algorithm's associated data structures as that algorithm executes (Fig. 3). A Generalized List Animation Tool (GLAT) [4] displays the contents of various list structures during the search. The user may also choose to examine current heuristic values for cities on these lists (Note: the heuristic value, or f-value, for a city is the estimated cost of including that city as the next step in a path from the start city to the goal city).

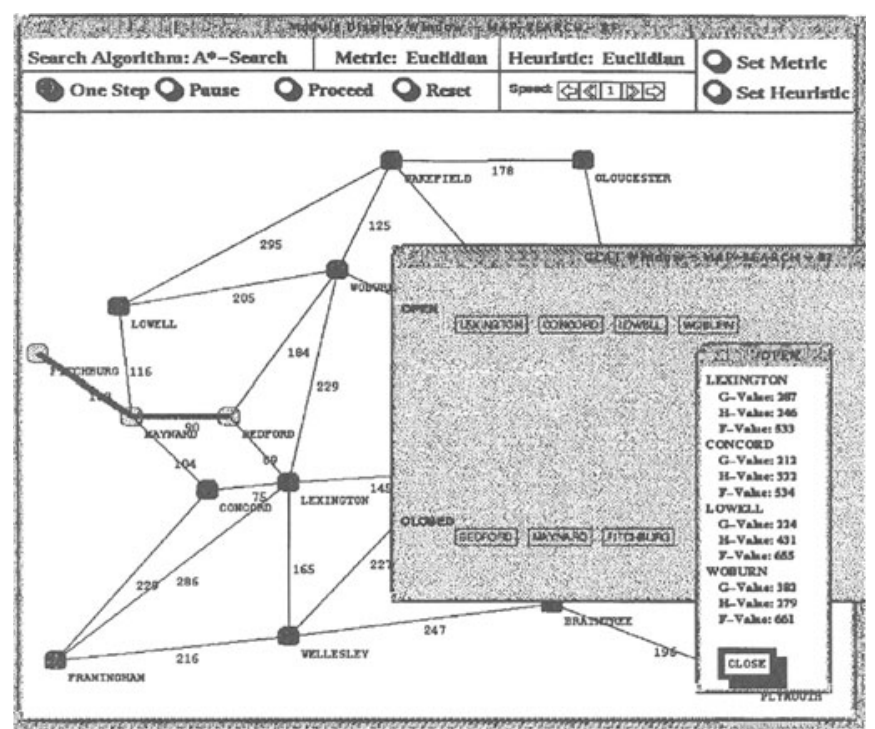

Fig. 3 The Map-Search module showing the detailed displays

\section{The theorem proving modules.}

The theorem proving modules do not provide an environment for experimental exploration; rather they provide dynamic graphical illustrations of how the theorem proving algorithms work and interact. 
The theorem proving module set includes the following modules:

- The Clausification Module translates a predicate logic formula to conjunctive normal form. The formulas which are output from this module, may be used as input to the Resolution-Proof-Procedure module.

- The Unification Module finds the Most General Unifier for two input clauses.

- The Binary Resolution module provides a step-by-step illustration of an application of the resolution inference rule.

- The Resolution Proof Procedure module determines whether a goal is derivable from a set of premises in conjunctive normal form. The module displays a proof tree if the goal is derivable (Fig. 4). Each step in the module recaptures a proof procedure step and allows the user to see how the resolution inference rule achieves the desired intermediate result. The user is invited to activate the Binary Resolution Module concurrently when the binary resolution algorithm is called.

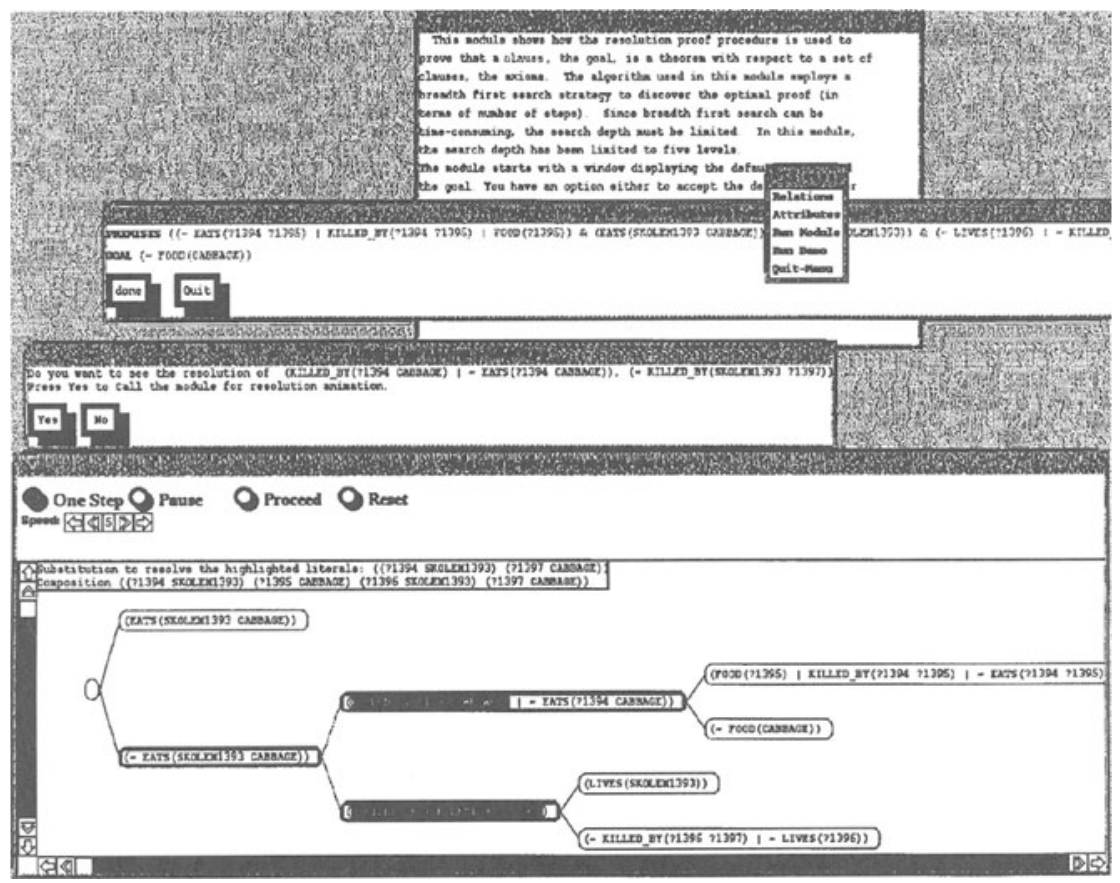

Fig. 4 The Resolution-Proof-Procedure model 


\section{FLAIR BASED LABORATORY ASSIGNMENTS}

Three nonprogramming laboratory assignments designed to be used with FLAIR are described in this section. None of the assignments requires that the student has mastered a programming language. The assignments include a two-hour structured or 'closed' laboratory assignment on the Best First (BF) and $\mathrm{A}^{*}$ heuristic search algorithms, a step-by-step tour or 'script' for the theorem proving modules, and a project in which teams of students design and build a conceptual map. ('Closed' or 'structured' labs refer to laboratory classroom assignments supervised by an instructor.)

\section{The heuristic search assignment}

This assignment has students explore differences between the $\mathrm{BF}$ and $\mathrm{A}^{*}$ heuristic search algorithms by examining and explaining differences in how the two algorithms solve specific search problems on a city map. Heuristic search algorithms use heuristic values (known as f-values) to find good or optimal paths between nodes in a graph. Heuristic search algorithms are distinguished, in part, by how they compute $\mathrm{f}$-values. Defining $\mathrm{g}$ as the estimated cost of the path from the start node to the current node, $h$ as the estimated cost of the path from the current node to the goal node and $\mathrm{f}$ as the sum of $\mathrm{g}$ and $\mathrm{h}$, then $\mathrm{BF}$ search estimates $g$ as 0 (so $f$ is just $h$ ) and $A^{*}$ estimates $g$ as the cost of the current best path from the start to the current node. Both algorithms keep track of partial paths from start to goal nodes and will switch to another path if the $f$-value for the end node on that path is better than the f-value for the current node.

The algorithms also differ in that BF only keeps track of the first best path found from the start node to the 'current' node, while $\mathrm{A}^{*}$ considers alternative paths through the current node selecting the best at each iteration. That is, if the $g$-value from the start node to the current node has been improved, $\mathrm{A}^{*}$ recomputes the f-values for all successors of the current node and re-examines (places on the OPEN list) all successors for which the f-value has been improved. Consequently, while BF may find a good path more quickly than $\mathrm{A}^{*}$, $\mathrm{A}^{*}$ finds an optimal path.

\section{Problem Specification}

For the first part of the laboratory assignment students are asked to attempt to specify a search problem for each of the following five cases:

1. A* finds a shorter path than $B F$.

2. $B F$ finds a shorter path than $A^{*}$.

3. $A^{*}$ and $B F$ find the same length path.

4. $\mathrm{A}^{*}$ examines more nodes than $\mathrm{BF}$. 


\section{BF examines more nodes than $\mathrm{A}^{*}$.}

Note that 2. and 5. are not possible to satisfy, but that failure to satisfy them can provide information about the algorithms.

For the second part of the assignment, students are asked to explain the observed differences between the algorithms.

The students were asked to produce two deliverables: a list of the search problems (start and end city) satisfying each of the five cases listed in part 1 and a few sentences answering the second part of the task.

\section{Experience with the assignment}

This assignment was used as the task for a pilot evaluation of the Map-Search Module [5]. Two computer science students who had had no experience with heuristic search algorithms worked collaboratively on the task for two hours after receiving a brief overview of the Module. The students used the main module display, the GLAT detailed display and the algorithm pseudocode extensively, and completed all of the first task and part of the second task within the allotted time.

The students' overall approach to the tasks suggests some pedagogical benefits of the learning environment provided by the module. On many occasions an episode of experimentation was followed by close examination of the pseudocode versions of the algorithms. In such cases visual observations of algorithm behaviour encouraged the students to carefully examine the pseudocode for explanations.

In several cases episodes of trial and error testing culminated in the formulation or refinement of hypotheses about the algorithms. These hypotheses were subsequently tested with additional search problems, leading to insights about the algorithms. It would have been nearly impossible for undergraduate students to perform this number of experiments using a paperbased method and still maintain a high degree of focus and motivation to continue.

The most striking event occurred midway into the second hour when the students noticed that the $\mathrm{A}^{*}$ algorithm unexpectedly 'jumped' to explore an alternative path from the source to the current node. This visual discovery prompted a re-examination of the pseudocode which led to the observation that, unlike $\mathrm{BF}, \mathrm{A}^{*}$ sometimes selects a new path from the start node to the current node. The discovery of this important difference between the algorithms can be attributed unequivocally to the animated visual environment provided by the module. 
The students continued to discuss the problem after the experiment had ended. Although their enthusiasm may have been due in part to a successful collaborative effort, it was also due in part to the dynamic module environment.

\section{A script for the theorem proving modules}

This assignment takes students on a step-by-step tour through FLAIR's theorem proving modules. One of the assignment's objectives is to provide an overview of how resolution theorem proving works. A second objective is to show students how to use FLAIR to test their own proofs.

\section{Problem Specification}

Students are asked to translate a simple logic problem expressed in English into a set of predicate logic formulas and then to enter these formulas into the Clausification module to derive a formula in conjunctive normal form (CNF). They are then directed to run the Resolution Proof Procedure module with the CNF formula as input.

\section{Experience with the script}

This script was used in the laboratory section of the undergraduate introductory AI course at Temple University during the Fall Semester, 1993. Most of our students had little difficulty mastering the translation of simple declarative English sentences into predicate logic formulas. In a few cases, however, the script revealed that the original set of predicate logic formulas resulting from the translation was incorrect. The primary benefit of this script was concrete reinforcement of the initial exposure to concepts students had through lecture and textbook.

\section{FLAIR project: building a conceptual map}

This project has students define and implement using the FLAIR navigator tool a text based conceptual map for a manageably small subdomain of AI not currently included in the shared repository at the FLAIR site. Building a conceptual map for a subject area is an excellent way to become familiar with that area, since it encourages authors to be precise and disciplined.

Lectures and/or textbook explanations of the knowledge domain provide useful background for the project, but lectures do not have to be comprehensive as students should be encouraged to use library and Internet resources to research the area to improve their conceptual maps.

\section{Problem specification}

The students are expected to map out the subject area as carefully as possible, defining the most primitive as well as the most general concepts and their relationships, and specifying any class hierarchies which may exist in the 
domain. We recommend that students work in small teams for approximately two weeks on this project after which they can present their maps to the class. The class can compare maps based on completeness of coverage, sensible definition of conceptual units and their relationships, adequacy of the examples used and efficacy of the conceptual network (the relations chosen to link concepts into a map). Although some maps may more successfully describe the subject area, it is important to remind students that there is no single correct way to go about doing this.

\section{Experience with the Project}

This project was used in the undergraduate AI course at Temple University in the Fall 1993 Semester with fuzzy logic as the subject area. Students were divided into teams of two or three. They had a brief lecture on fuzzy logic, but no coverage was given the topic in their textbook [6]. We were surprised by the competitive spirit which developed among teams and the amount of library research each team did as a consequence. When the projects were due the maps were brought up on separate machines in the laboratory, explained and then compared. One of the maps was sufficiently complete and clear to be incorporated (with some modifications) into the FLAIR repository to extend the conceptual map available to all users at the site, while the other maps suffered from deficiencies which proved educational.

\section{CONCLUSIONS}

Graphical and animated features of the FLAIR system permits the design of nonprogramming assignments to support the laboratory component of the introductory undergraduate AI course. Although we have not shown formally that students' performance is improved when they complete such assignments with FLAIR, we have noted informally that students find these assignments motivating. All three assignments ground abstract ideas discussed in lecture and textbook with concrete examples and with experimentation which may explain why students find such assignments engaging.

\section{ACKNOWLEDGEMENT}

This research has been supported in part through the Educational Infrastructure Program of the National Science Foundation (Grant \#CDA-9115254). 


\section{REFERENCES}

1. Chi, M.T.H. and Bassok, M. (1989) Learning From Examples via SelfExplanations, in Resnick, L.B.(ed.), Knowing, Learning, and Instruction, Lawrence Erlbaum Associates, New Jersey, pp. 337-360.

2. Ross, B.H. and Kennedy, P.T. (1990) Generalizing From the Use of Earlier Examples in Problem Solving. Journal of Experimental Psychology: Learning, Memory, and Cognition, 16, pp. 42-55.

3. Ingargiola, G., Hoskin, N., Aiken, R., Dubey, R., Wilson, J., Papalaskari, M.A., Christensen, M. and Webster, R. (1994) A Repository that Supports Teaching and Cooperation in the Introductory AI Course. ACM-SIGCSE Technical Symposium on Computer Science Education, pp. 36-40.

4. Webster, R., and Ross, P. (1991) Useful Artificial Intelligence Tools-A Review of heuristic search methods. IEEE Potential Journal, Oct. 1991, pp. 5154.

5. Wilson, J., Katz, I., Ingargiola, G., Aiken, R. and Hoskin, N. (1995) Student's Use of Animations for Algorithm Understanding. Short Paper, CHI '95, Denver, Colorado, May 1995.

6. Rich, E. and Knight, K. (1991) Artificial Intelligence, 2nd

Edn, McGraw-Hill. 